\title{
07 - Strong acute-stress impairs cortical synaptic plasticity in the amygdala
}

\author{
Sofia Rodrigues, Natália Madeira, Rosalina Fonseca
}

\section{Introduction}

Learning is a key process that allows indi vidual s to adapt to the environment. Consider able evidence indicates that new $m$ em ories are form ed upon a large netw ork of pre-existing knowledge. This implies that learning is highly influenced by previous experience and that previous consolidated memories can be reactivated during new learning. This is particularly relevant in the context of traumatic memories, in which the form ation of a particular association leads to a disruptive behaviour. In post-traum atic stress dis order (PTSD) an over-generalization and hy per-reactivity of fear responses is observed after expos ure to a traumatic event. There is clear evidence that subjects with PTSD show not only a behaviour al sensitization to stress and an over-generalization to neutral stimuli but also an impair ed extinction of the initial association established during the traumatic event. This suggests that PTSD may alter the normal dynamic flow of memory. The link between endocannabinoid signalling, the acquisition of fear memories and stress is well established. We haverecent data suggesting that CB1R activation restricts synaptic cooper ation and competition in the am ygdala by reducing thalamic synaptic activation. Also, we have preliminary evidence that CB1R are differently expressed between thal amic and cortical presynaptic boutons. These observations, together with a recent report that endocannabinoid synthesis is sensitized by previous negative events, support the hypothesis that endocannabinoid system function as a "gatekeeper" restricting fear-memory acquisition. We aim to 1) using an animal model of PTSD, as sess the properties of synaptic plasticity in the thal amic and cortical affer ents to the lateral amygdala; 2) as sess whether modulation of the cannabinoid signalling regulates the devel opment of a PTSD phenotype.

\section{Results}

We use a combination of behavioural analysis and el ectrophysiology to assess the behavioural profile of the animals and synaptic properties in the am ygdala. We analys ed the individual scores of the animals in two time points (Figure 1) and defined a behavioural profile according to the deviation to the control group.
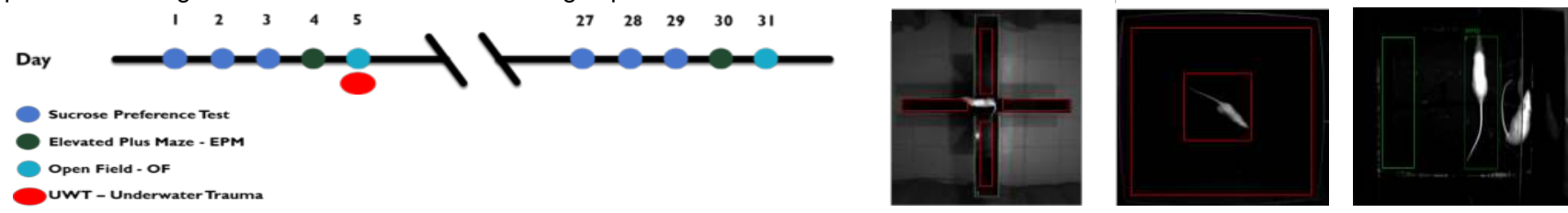

We observed that exposing animals to a single high-stress or (UWT) lead to the development of an anxiety profile that is expres sedin a lower exploration of the open arm of the el evated plus maze (EPM - B) as well as alower time spent in the inner-zone of the open field arena (OF - A; Figure 2). No change was observed in the sucrose-preference test suggesting that a depress ed-like phenotype is not present. In a sel ected group of animals we perform ed an additional behaviour test, the social-prefer ence test an observed an impairment in stressed exposed animals (C).

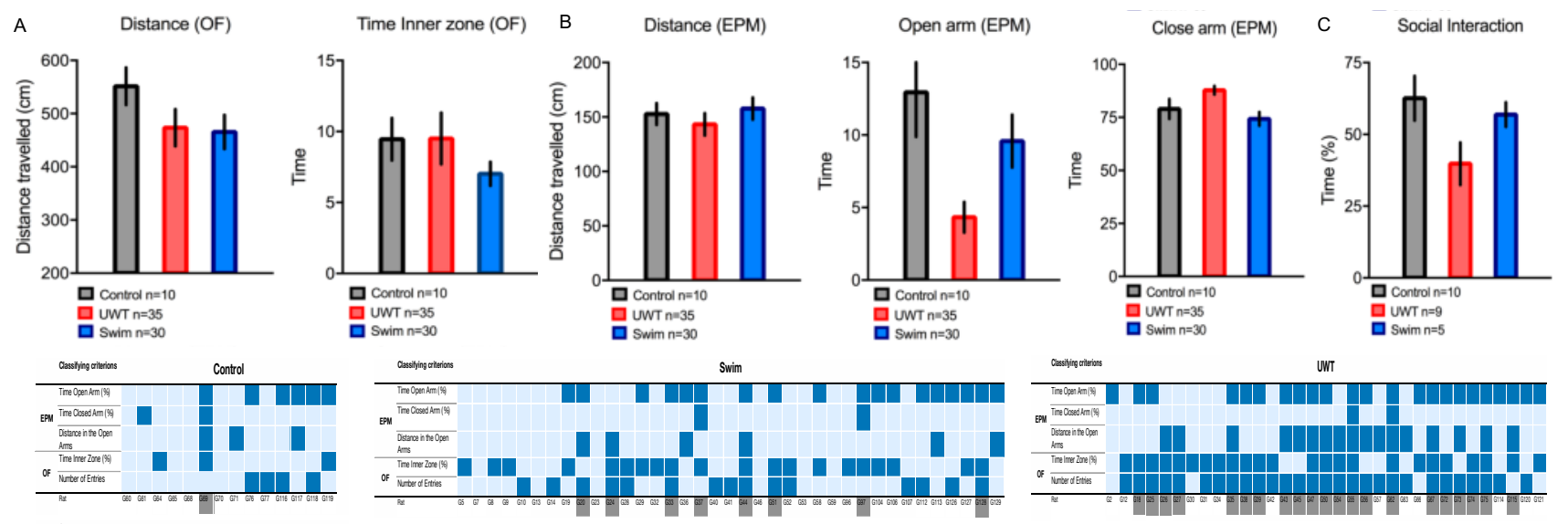

Patch -clamp recor dings of am ygdala pyramidal cells (LA) showed that cells from stressed anim als are more excitable (lower actionpotential threshold) al though their resting membrane potential is unchanged (Figure 3, A/B). WE also observed that cortical LTP in unpaired in stressed exposed animals (Figure $4-C / D$ ), as compared with Swim (E/F) and control animals (A/B).

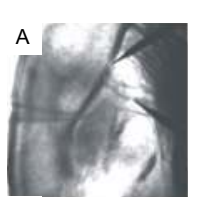

B
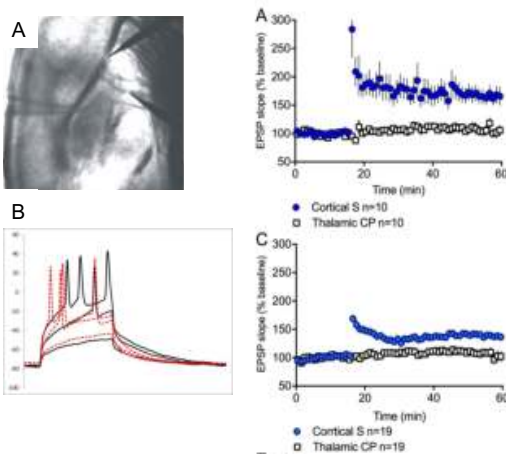

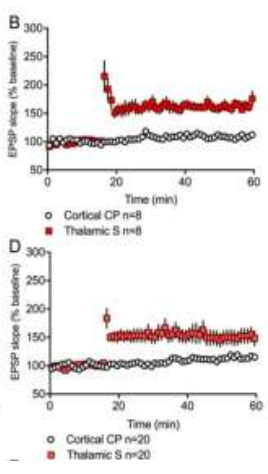

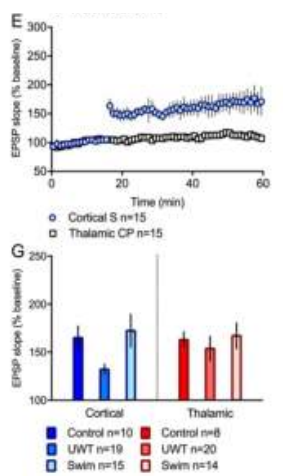

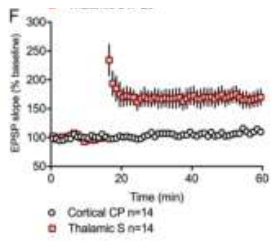

Our results show that not all animals exposed to the UWT stressor develop a PTSD phenotype, evident in the individual profile. We will further address whether CB1R signalling in involved in resilience.

This work is supported by grants from the Portuguese Science Foundation (FCT - IF/01359/2014 e PTDC/NE U-SCC/3023/2014) and by a NARS AD Grant from the Brain and Behaviour Research Foundation. Copyright @ 2019 Rosalina Fonseca, Cellular and Systems Neurobiology, CEDOC - NOVA Medical School, Lisbon Portugal rosalina.fonseca@ nms. unl. pt 